\title{
Arbeitsbedingungen und Mitbestimmung in den neuen Arbeitsverwaltungen
}

Die institutionelle Reform der Arbeitsmarktpolitik wird mehrheitlich (in 356 Städten und Gemeinden) durch Arbeitsgemeinschaften (ARGEn) mit einer Kooperation zwischen Bundesagentur für Arbeit und Kommunen umgesetzt. Im Optionsmodell übernehmen dagegen 69 Modellkommunen alle SGB-II-Leistungen eigenständig und betreuen die Langzeitarbeitslosen alleinverantwortlich. Die Institutionalisierung der Options- und Kooperationsmodelle hat auffallende Konsequenzen: Die Personalvertretungen können nur unter Unsicherheit arbeiten. Mitbestimmung und damit Einfluss auf die ohnehin belastenden Arbeitsbedingungen der Beschäftigten wird erschwert. $^{1}$

\section{Arbeitsmarktpolitische Institutionen als politisches Experiment}

Die arbeitsmarktpolitischen Institutionen des Options- und Kooperationsmodells beruhen auf einem langwierigen Interessenclearing zwischen unterschiedlichen Akteuren aus Politik, Städtetag, Landkreistag, Bundesagentur für Arbeit u.a. Die heutige Organisation der Trägerschaft ist das Resultat eines politischen Einigungsverfahrens mit folgenschweren institutionellen Kompromissen:

(1) In den Einrichtungen des Kooperationsmodells existiert ein breiter Gestaltungsspielraum bei der praktischen Umsetzung dieses Konzepts. So werden die ARGEn auf der lokalen Ebene durch private oder öffentlich-rechtliche Verträge (wie $\mathrm{GmbH}, \mathrm{GBR}$ oder Anstalt des öffentlichen Rechts) eingerichtet, in die die Bundesagentur für Arbeit und die Kommunen Personal abgeben. $\mathrm{Zu}$ diesen untypischen Mischverwaltungen und den personalbzw. arbeitspolitischen Voraussetzungen und Auswirkungen existieren hierzulande allerdings keine Vorerfahrungen.

(2) In den Optionskommunen bildet sich zwar ebenfalls eine Vielfalt von Organisationsvarianten heraus, wenn die neuen Einrichtungen als kommunale Ämter oder Abteilungen, kommunale Eigenbetriebe oder Gesellschaften installiert werden (vgl. ausführlich Bertelsmann Stiftung 2006).
Dennoch ist die institutionelle Unsicherheitszone in den Optionskommunen mit einer ungeteilten Trägerschaft und einheitlichen Personal- und Ressourcenverantwortung deutlich geringer ausgeprägt als in den ARGEn.

Das Resultat dieser "Versuchsanordnung" ist offen und damit die Frage, welche strukturellen Modellentscheidungen zu erwarten sind. Die Architektur wird sich aber zweifellos verändern. Dazu trägt inzwischen maßgeblich das Urteil des Bundesverfassungsgerichts (vom Dezember 2007) bei, das die Mischverwaltung ARGE, diese Gemeinschaftseinrichtung von Bund und Kommune, für verfassungswidrig erklärt hat. Allerdings wurde dem Gesetzgeber eine Entscheidungsfrist bis Ende 2010 eingeräumt. Die Debatte über den institutionellen Pfad der Arbeitsmarktreform bleibt somit in Gang und damit vor allem die Aussicht unklar, unter welchen Bedingungen die Beschäftigten ihre Aufgaben zukünftig wahrnehmen und die Personalräte ihre Interessen vertreten werden. Dies lenkt den Blick auf die Fragen, wie derzeit die Personalvertretungen in den Organisationen mit welchen Interessenvertretungsstrategien mitbestimmen und unter welchen Arbeitsbedingungen sowie Arbeitsbelastungen die Beschäftigten ihre arbeitsmarktpolitischen Aufgabenstellungen erfüllen. Gegenwärtig unterscheiden sich jedenfalls nicht nur die Organisationsprobleme und Arbeitsbedingungen, sondern auch die Handlungschancen bzw. Mitbestimmung der Personalvertretungen im Options- und Kooperationsmodell.

\section{Personalentwicklung unter Organisationsproblemen}

Die „Begehung“ von Optionskommunen im Rahmen von Fallstudien schafft Transparenz über typische Implementationsphasen, welche die neuen kommunalen Organisationseinheiten durchlaufen. In den Kommunen, die als Optionskommunen zugelassen wurden, versprachen sich

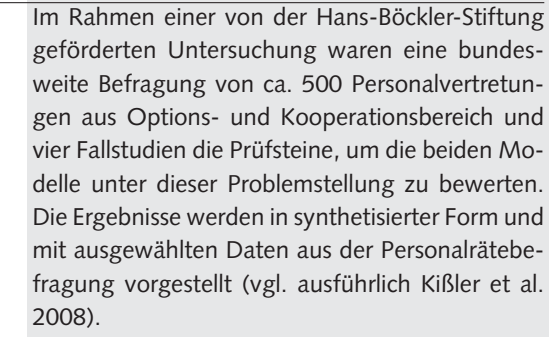
geförderten Untersuchung waren eine bundesweite Befragung von ca. 500 Personalvertretungen aus Options- und Kooperationsbereich und vier Fallstudien die Prüfsteine, um die beiden Modelle unter dieser Problemstellung zu bewerten. Die Ergebnisse werden in synthetisierter Form und mit ausgewählten Daten aus der Personalrätebefragung vorgestellt (vgl. ausführlich Kißler et al. 2008)

Ralph Greifenstein, Dipl. Soz., freiberuflicher Wissenschaftler und Berater. Arbeitsschwerpunkt: Industrielle Beziehungen. e-mail: RGreifenstein@aol.com Leo Kißler, Dr. Dr., Professor für Soziologie an der Philipps-Universität Marburg. Arbeitsschwerpunkt: Organisations- und Partizipationsforschung. e-Mail: kissler@staff.uni-marburg.de Elke Wiechmann, Dr., Soziologin, freiberufliche Wissenschaftlerin und Beraterin. Arbeitsschwerpunkte: Arbeitsorganisation, Chancengleichheit. e-Mail: elke.wiechmann@t-online.de 
Kommunalpolitiker bessere lokale Handlungs- und Gestaltungsmöglichkeiten, um die (Langzeit-) Arbeitslosigkeit in kommunaler Eigenregie effizient $\mathrm{zu}$ managen. Nicht zu übersehen ist aber auch eine andere Absicht. Gemeint ist die Perspektive, kommunales Personal, das im Zuge der Verwaltungsrationalisierung freigesetzt wird, in die neuen Organisationseinheiten zu versetzen. Dafür bietet das Optionsmodell, der Aufbau einer neuen kommunalen Organisationseinheit, durchaus eine günstige Gelegenheit.

Bevor die Betriebsfähigkeit der neuen Einrichtungen gewährleistet werden konnte, waren allerdings unter beachtlichem Arbeitseinsatz erhebliche Implementationsprobleme zu bewältigen. Nach Überwindung der Startschwierigkeiten reift inzwischen der SGB-II-Betrieb in den Optionskommunen. Das integrierte System von materieller Absicherung, Beratung und Betreuung wird funktionsfähiger. Mit Hilfe der anspringenden Konjunktur stellen sich auch arbeitsmarktpolitische Erfolge ein. Viele arbeitsmarktnahe Kunden werden in Arbeit gebracht. Die arbeitsmarktferne Klientel mit multiplen Vermittlungshemmnissen verbleibt dagegen mehrheitlich in den neuen SGB-II-Einrichtungen der Optionskommunen.

In den meisten Optionskommunen fehlt zudem noch eine eingeübte Praxis im Bereich der Arbeitsmarkt- und Beschäftigungspolitik, was allerdings durch die Personalrekrutierungsstrategien teilweise kompensiert wird. Die Optionskommunen nahmen ergänzend zum kommunalen Personaltransfer durchgängig (in der Regel befristete) Neueinstellungen aus unterschiedlichen Branchen bzw. Berufsgruppen vor. Die Umsetzungen und Neueinstellungen führten zu einer gemischten Personalstruktur in den neuen Organisationseinheiten mit dem Vorteil, dass besonders die neuen Mitarbeiter/innen Qualifikationen bzw. Arbeitsmarktkompetenzen einbringen, die in der Kommunalverwaltung fehlen. Zwar kann aufgrund dieses Personalmix in den Optionskommunen durchaus von einem "Zusammenprall der Kulturen“ gesprochen werden (Knuth et al. 2007, S. 76). Aber, unter der einheitlichen Personalverantwortung eines Arbeitgebers ziehen die Beschäftigten (im Vergleich zu den ARGEn) eher „an einem Strang“ und glätten interkulturelle Divergenzen, um ihr Optionsmodell zu stabilisieren. Das Arbeitsverhältnis bietet den Neueingestellten trotz der Befristungen auch eine berufliche Entwicklungschance. Die befristet Beschäftigten verwenden erhebliche Energien darauf, noch besser zu werden. Sie versprechen sich vom Optionsmodell, vorausgesetzt es besteht weiter, eine stabile Arbeitskarriere in einem neuen Aufgabenfeld. Die Aussichten im Falle einer politischen Rücknahme des Optionsmodells wären für befristet wie unbefristet Beschäftigte gleichermaßen ungünstig: den Einen fehlt die langfristige Arbeitsplatz- und Existenzsicherheit, den Anderen eine qualitative Rückkehrperspektive in die Kommunalverwaltung, d. h. ein qualifikations- und laufbahngerechter Arbeitsplatz.

Die Institutionalisierung der ARGEn mit gemeinsamer Aufgabenwahrnehmung von Bund und Kommunen ist sowohl während der Implementation als auch im Konsolidierungsverlauf ein komplexeres Vorhaben. Folgende Funktionsstörungen bzw. Strukturprobleme sind hervorzuheben:

(1) Die gemeinsame Trägerschaft zwischen Agentur für Arbeit und Kommunen ist ein diffiziles Geschäft. Die Bearbeitung von konfligierenden Interessen im Institutionalisierungsprozess gleicht häufig einer Verhandlung zwischen David und Goliath und absorbiert erhebliche Energien und Ressourcen.

(2) Die Personalstruktur der ARGEn kennzeichnet eine Mischung aus kommunalen und Agenturbeschäftigten. Beide Beschäftigtengruppen bringen höchst unterschiedliche Qualifikationen, Kompetenzen und Arbeitskulturen in die neuen Institutionen ein, die erst aufeinander abzustimmen sind.

(3) Eine hinreichende Personaldecke ist eine wichtige Voraussetzung für die Betriebsfähigkeit der ARGEn. Allerdings starteten die Trägereinrichtungen zumeist mit personeller Unterbesetzung. Die Schwerpunkte der anschließenden Personalaufstockung liegen vor allem in den Hauptaufgabenbereichen Leistungsgewährung, Arbeitsvermittlung und Fallmanagement. Eine weiteres personalpolitisches Kennzeichen ist der hohe Anteil von befristet Beschäftigten.

Einige Organisationsprobleme sind demnach durchaus vergleichbar zu den Optionskommunen, andere kommen in den
Mischverwaltungen allerdings hinzu. Es sind aber nicht nur die institutionellen Strukturprobleme der ARGEn, die die Betriebsfähigkeit behindern, sondern zugleich eine Schwachstelle in den Arbeitsbeziehungen. Auffällig sind die Mitbestimmungsschwierigkeiten, welche die Arbeitsbeziehungen belasten und die Gestaltung der Arbeitsbedingungen beeinträchtigen.

\section{Mitbestimmungsdefizit im Kooperationsmodell}

Die Arbeitsbeziehungen in den ARGEn kennzeichnen unklare und experimentelle Mitbestimmungsstrukturen. In den Mischverwaltungen von Bund und Kommune fehlt zumeist eine gemeinsame Interessenvertretungspolitik. Die Personalräte sind je nach Vertragsgestaltung der ARGEn für das Personal der jeweiligen Stammhäuser zuständig, oder aber zugewiesene Beschäftigte verlieren in der ARGE ihr aktives und passives Wahlrecht. Die Personalvertretungen der Herkunftsorganisationen sind daher zu einem guten Teil mit ihrer eigenen Interessendurchsetzung beschäftigt. Die Mitbestimmung verliert an Substanz, denn die Personalräte können weder ihre Gestaltungs- noch Schutzmacht für die Beschäftigten ausreichend aktivieren. Wenn verhandlungsmächtige Interessenvertretungen fehlen, haben die Beschäftigten geringere Chancen, ihre Interessen zur Gestaltung der Arbeitsbedingungen in den ARGEn gemeinsam durchzusetzen. So konnten beispielsweise simple Dienstvereinbarungen zur Genehmigung von Dienstreisen in einer der untersuchten ARGE nicht abgeschlossen werden, da eine für alle Mitarbeiter zuständige Personalvertretung fehlte.

Dieser interessenvertretungspolitische Notstand führt dazu, dass sich in den ARGEn mittlerweile Interessenvertretungsformen mit Personalräten beider Leistungsträger etablieren. In einer Kommune wurde beispielsweise ein Betriebsbeirat für die ARGE gegründet, bestehend aus den Personal- und Betriebsräten der beteiligten Organisationen (Arbeitsagentur, Kommune und Regionale Personalentwicklungsgesellschaft). Seine Rechte sind aber bei weitem nicht mit den personalvertretungsrechtlichen bzw. betriebverfassungsgesetz- 


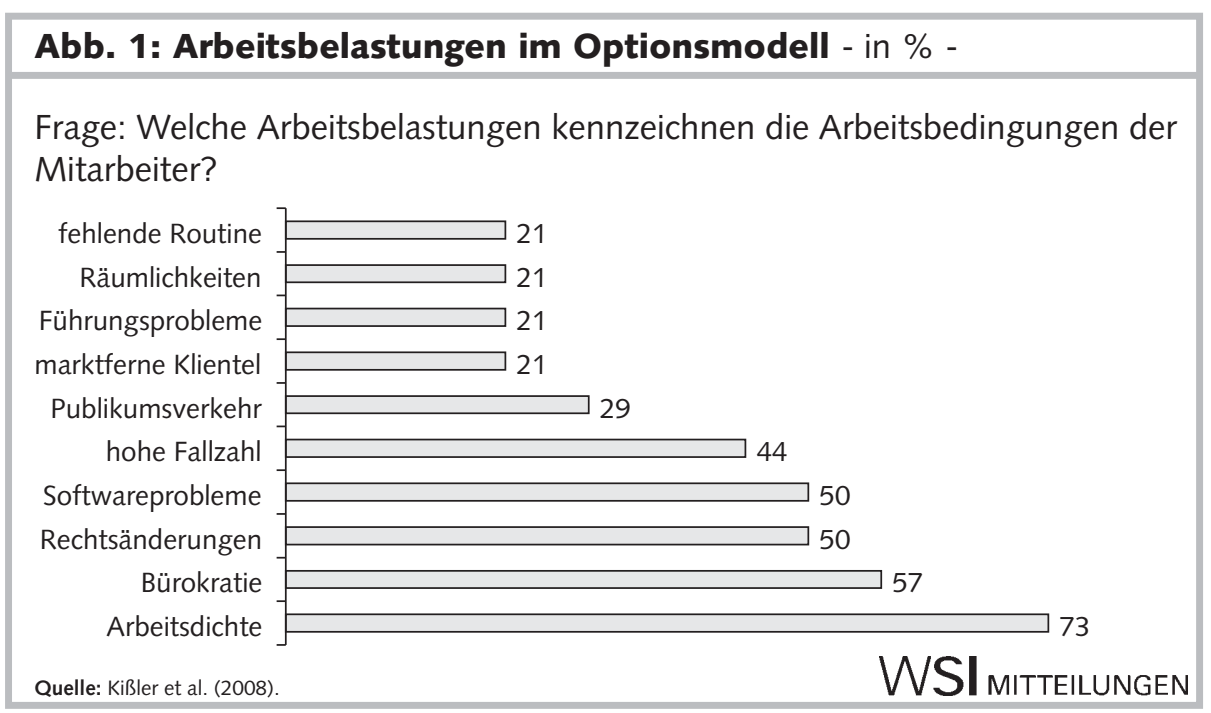

lichen Mitbestimmungsregelungen vergleichbar, sondern bewegen sich in einer Grauzone jenseits rechtlich abgesicherter Arbeitnehmerbeteiligung. Die Personalund Betriebsräte können die unterschiedlichen Eingruppierungen der Mitarbeiter aus Arbeitsagentur und Kommune, Tätigkeitsinhalte, Leistungsbeurteilungen oder Arbeitszeiten nicht regulieren bzw. keinen einheitlichen Standard innerhalb der ARGE schaffen.

In einer anderen ARGE wählen die Beschäftigten einen eigenständigen, rechtlich im Prinzip aber ebenfalls nicht abgesicherten Personalrat. Auch ohne einwandfreie rechtliche Absicherung soll pragmatisch versucht werden, die Interessen der Beschäftigten vor Ort in der ARGE durchzusetzen. Dazu sind auch regelmäßige $\mathrm{Zu}$ sammenkünfte mit dem Personalrat der Arbeitsagentur und der Kommune (gemeinsames Personalratsgremium) vorgesehen, um die Handlungsmöglichkeiten abzusprechen. Das setzt allerdings voraus, dass die Geschäftsleitung der ARGE diese Variante von Interessenregulierung stets, also auch in Konfliktfällen, anerkennen wird.

Solche Versuche zeigen, wie ausprobiert wird, die Vertretungslücke zu schließen und eine Mitbestimmung zu institutionalisieren, wenn auch mit verminderter Durchsetzungskraft. Dadurch geraten die Personalräte allerdings in das Dilemma, ihre Personalvertretungsstrategie ohne rechtssicheren Akteurstatus umsetzen zu müssen, um die Arbeitsbedingungen der Beschäftigten zu verbessern. Die vertretungsgeschwächten Personalräte können im Kooperationsmodell auch weitaus weniger als Ko-Manager agieren als ihre Kollegen in den Optionskommunen. Die Personalräte nehmen die Interessenvertretungsarbeit im Kooperationsmodell daher als eine große Herausforderung wahr.

Die Interessenvertretung im Optionsmodell stützt sich demgegenüber auf eine bewährte Mitbestimmungspraxis. Die Personalratsarbeit im Optionsmodell folgt der normalen Interessenvertretungsarbeit in einer Kommunalverwaltung und ihren geregelten Arbeitsbeziehungen. Ohne den Personalrat wird das Optionsmodell in der kommunalen Verwaltungspraxis nicht umgesetzt. Die lokalen Unterschiede, d.h. wie die Personalvertretung mitbestimmt, wird lediglich von der verwaltungsspezifischen Kultur der Arbeitsbeziehungen zwischen Führungsspitze und Interessenvertretung bzw. dem lokalen Personalratstyp beeinflusst. Die Personalräte wurden entweder schon vor der Optionsentscheidung oder zumindest kurz danach am kommunalen Optionsvorhaben beteiligt. Die Konzeptions-, Umsetzungs- und Korrekturpartizipation der Interessenvertretungen ist regelmäßig gefragt.

Diese Einsichten zeigen: Die Gestaltungschancen der Interessenvertretungen sind im Options- und Kooperationsmodell unterschiedlich verteilt und damit auch die Möglichkeiten, Arbeitsbedingungen zu verbessern und Arbeitsbelastungen zu verringern. Die Arbeitsbelastungen sind aber in beiden Modellen nicht zu unterschätzen und erfordern eine gute Interessenvertretung, wie die folgenden Ergebnisse einer bundesweiten Personalrätebefragung belegen.

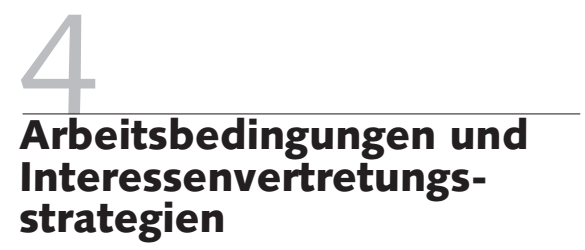

Die institutionelle Neustrukturierung der Arbeitsmarktpolitik führt in beiden Modellen zu auffallenden Arbeitsbelastungen. Dabei spielt auch die Binnenorganisation, d.h. die Organisation der Leistungserbringung eine Rolle, z. B. in der Frage, ob es sich eher um einen spezialisierten oder generalisierten Fallmanagementansatz handelt und die Vermittlung auf den Arbeitsmarkt ins Fallmanagement integriert ist oder nicht (vgl. zu dieser Organisationstypologie ausführlich ISG 2007, S. 69ff.)

Zunächst wird der Blick auf das Optionsmodell gerichtet (Abbildung 1). In den neu eingerichteten kommunalen Organisationseinheiten werden die Beschäftigten unter verschiedenen Arbeitsbelastungen tätig:

Die Institutionalisierung des Optionsmodells hat unterschiedliche Folgen für die Mitarbeiter/innen, wie die Gewichtung der Arbeitsbelastungen zeigt: Das Arbeitspensum ist unter erhöhtem Zeitdruck bzw. starker Leistungsverdichtung zu bewältigten. Die Dienstleistungs- und Servicequalität wird vor allem durch unterschiedliche Rahmenbedingungen zur Kundenbetreuung beeinträchtigt. So ist eine defizitäre EDV ebenso kontraproduktiv wie ein „Papierkrieg", der Arbeits- und Betreuungszeit absorbiert. Die fortwährend veränderte SGB-II-Gesetzgebung bietet den Beschäftigten darüber hinaus noch zuwenig Rechtssicherheit. Hervorzuhebende Arbeitsbelastungen sind aber auch ein noch zu hoher Betreuungsschlüssel und der starke Publikumsverkehr. Diese belastenden Arbeitsbedingungen müssen die Beschäftigten mit dem Risiko akzeptieren, dass sie ihre Arbeitsproduktivität durchaus überstrapazieren.

Innerhalb der Belegschaften unterscheiden sich die Arbeitsbelastungen. Die Fallstudien in Optionskommunen ergaben, dass die institutionelle Bewährungsprobe besonders die Fallmanager mehrfach herausfordert:

(1) Ein vermittlungsorientiertes innerbetriebliches Controlling und eine Kategorisierung der Kunden nach ihrer prognostizierten Vermittelbarkeit bzw. Arbeits- 
marktnähe erzeugen einen Erfolgsdruck, der zur Konzentration auf die „passenderen“ Kunden verführt, nicht immer explizit, aber zumindest in den Köpfen der Fallmanager. Der Service für schwierigere Kunden mit hohem Betreuungsaufwand wird infolge zum persönlichen beruflichen Konfliktfeld, da (in der Wahrnehmung der Fallmanager) der noch zu hohe Betreuungsschlüssel sowie ungünstige Vermittlungschancen ein „Fördern“ kaum erlauben.

(2) Fallmanager und Arbeitsvermittler sind zudem gefordert, sich beruflich weiterzuentwickeln, da ihnen teilweise ausreichende Arbeitsmarktkenntnisse bzw. -erfahrungen fehlen, um die Arbeitsuchenden mit eingeübten Integrationsstrategien auf dem lokalen und überregionalen Arbeitsmarkt unterzubringen. Diese Kenntnisse können im Rahmen beruflicher Weiterbildung nur begrenzt „seminaristisch“ vermittelt werden, sondern setzen längere praktische Lernprozesse in den optierenden Kommunen voraus.

(3) In den untersuchten Optionskommunen wird zwar kein lupenreiner generalisierter Fallmanagementansatz gefahren, sondern z. T. übernehmen auch separate Organisationseinheiten bzw. Akteure die Vermittlung auf den ersten Arbeitsmarkt. Allerdings wird die Arbeitsvermittlung in einem Fall stark an das Fallmanagement gekoppelt und die Fallmanager sollen auch an ihrer Integrationsleistung gemessen werden. In einer anderen Kommune wird keine Trennung vorgenommen, sondern der Fallmanager ist sowohl Vermittler auf den Arbeitsmarkt als auch Berater der Betreuungskunden. In beiden Konzeptionen markiert der Vermittlungs- bzw. Integrationsdruck aber eine Arbeitsbelastung der Fallmanager.

Die Mitarbeiter/innen der Leistungsgewährung bewegen sich ebenfalls in einer Unsicherheitszone. Diese Beschäftigten sind vor allem gefordert, die rechtlichen Innovationen bzw. Korrekturen im SGB II mit zu vollziehen. Diese (belastende) Aufholjagd mit der SGB-II-Gesetzgebung ist nur durch regelmäßige Schulungen zu gewinnen, die den Beschäftigten höhere Verfahrenssicherheit bieten.

Nun der Blick auf die Arbeitsbedingungen in den ARGEn (Abbildung 2): Die Beschäftigten in den ARGEn stellen sich ge-

Abb. 2: Arbeitsbelastungen im Kooperationsmodell - in \% -

Frage: Welche Arbeitsbelastungen kennzeichnen die Arbeitsbedingungen der Mitarbeiter?

$$
\begin{array}{r}
\text { fehlende Routine } \\
\text { unzureichende Räume } \\
\text { Publikumsverkehr } \\
\text { Rechtsänderungen } \\
\text { marktferne Klientel } \\
\text { Bürokratie } \\
\text { Software } \\
\text { hohe Fallzahl } \\
\text { Arbeitsdichte }
\end{array}
$$

Quelle: Kißler et al. (2008)

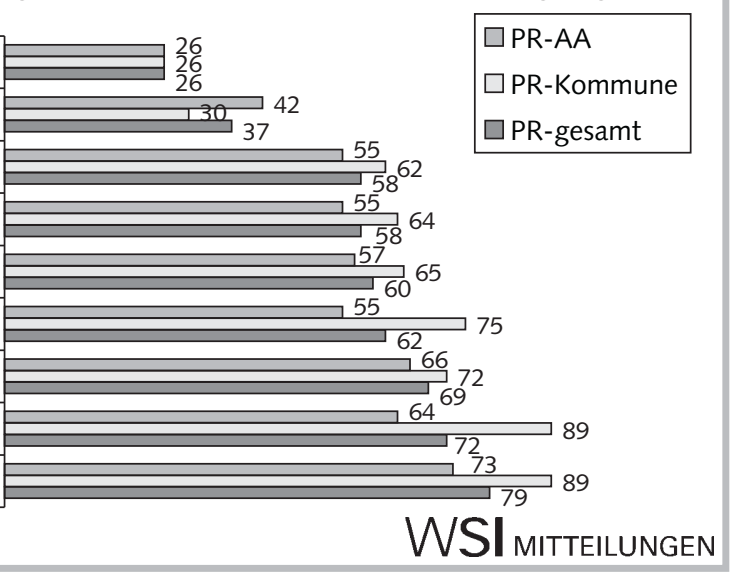

nauso wie ihre Kollegen in den Optionskommunen unterschiedlichen Arbeitsbelastungen, die wie folgt von den Personalräten aus Arbeitsagentur (PR-AA) und Kommune (PR-Kommune) gewichtet wurden:

Die Arbeitsverdichtung, hohe Fallzahlen, Softwareprobleme und der bürokratische Aufwand zählen auch in den ARGEn zu den zentralen Arbeitsbelastungen. Über diese vier stärksten Belastungen hinaus verunsichern die fortwährenden Rechtsänderungen. Ebenso belastend ist aber eine arbeitsmarktferne Klientel mit geringen Vermittlungschancen.

Personalräte aus Arbeitsagentur und Kommune bewerten die Arbeitsbelastungen aber durchaus unterschiedlich. Einerseits heben kommunale Personalräte stärker die „Bürokratie“ hervor. In den ARGEn sind die kommunalen Beschäftigten ungeübt im Umgang mit einem zentralistisch normierten Regelsystem (der BA). Andererseits zeigt die stärkere Akzentuierung der Belastungsfaktoren „hohe Fallzahlen“ und „Arbeitsdichte“, dass die kommunalen Beschäftigten zugleich mit einer unerwarteten Arbeitsintensität konfrontiert werden. Dahinter verbergen sich nicht nur neue Qualifikationsanforderungen, sondern auch eine unklare Definition des Fallmanagements in der Implementationsund Konsolidierungsphase. So herrscht in einer untersuchten ARGE die Wahrnehmung vor, dass das (spezialisierte) Fallmanagement mit der Vermittlung konkurriert. Die „innerbetriebliche Konkurrenz“ ist aber unausgewogen, da im Fallmanagement die Regelung verschiedener Problemlagen im Vordergrund steht und der Erfolg der Fallmanager, ihr Arbeitsergebnis (Integrationsquote) kaum transparent darstell- bar ist. Dieser Legitimationsdruck führt zu einer zusätzlichen Arbeitsbelastung. Die Fallstudien zeigen in den ARGEn zudem: Ausbaufähig sind die Kenntnisse der Fallmanager und Arbeitsvermittler über den lokalen und regionalen Arbeitsmarkt. Zusätzlich fehlen den Beschäftigten in diesem Arbeitsbereich Beratungskompetenzen und psychosoziale Betreuungsfähigkeiten, um die schwierigen sozialen Problemlagen der Klientel zu bewältigen. Nicht zu unterschätzen ist aber auch der Qualifizierungsbedarf in der Leistungsgewährung. Vorrangige Maßnahmenfelder sind dort insbesondere Qualifizierungen im Bereich „Recht“ und „Umgang mit Publikum“.

Die Verringerung der aufgezeigten Arbeitsbelastungen markiert im Options- wie Kooperationsmodell eine Personalratsaufgabe. Die Brennpunkte der Personalratsarbeit während der Einrichtung der neuen Institutionen zeigen, auf welchen Beteiligungsfeldern die Personalvertretungen zu allererst aktiv wurden (Abbildung 3).

Akzeptable Arbeitsbedingungen mit ausreichender Mitarbeiterbeteiligung tragen zweifellos zur Stabilisierung neuer Organisationseinheiten bei. In den Optionskommunen wurden die Beschäftigten zwar beteiligt, aber nicht immer in befriedigendem Maße. Während der Konzeption und Implementation wurde die Mitsprache am Arbeitsplatz daher auch auf Initiative der Personalräte gestärkt, um die Arbeitsbedingungen und den Arbeitsablauf zu verbessern. Zudem achteten die Personalräte auf eine hinreichende Personalausstattung. Auch die Leistungsgerechtigkeit wurde zur Personalratsaufgabe, damit die Arbeitsentgelte den Aufgabenstellungen und Herausforderungen in den 


\section{Abb. 3: Strategische Personalratsarbeit während der Implemen- tationsphase im Optionsmodell - in \% -}

Frage: Wie bedeutsam waren bisher folgende Felder strategischer Personalratsarbeit im Optionsmodell?

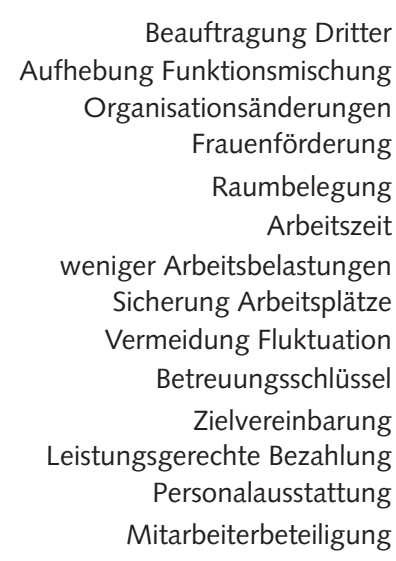

Quelle: Kißler et al. (2008).

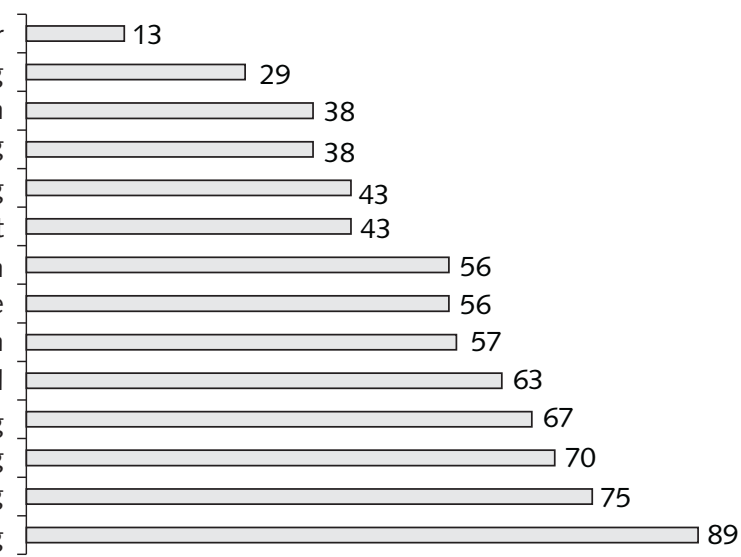

WSI MITTELLUNGEN neuen kommunalen Verwaltungseinrichtungen entsprechen.

In der Implementationsphase waren die Personalräte darüber hinaus gefordert, darauf hinzuwirken, dass den Beschäftigten realistische, erreichbare Ziele gesetzt werden. Weitere Personalratsziele kommen dazu: Arbeitsplatzsicherheit, eine zu bewältigende Anzahl an Kunden sowie Arbeitsbedingungen, welche die normale Personalfluktuation nicht erhöhen. Auf der Suche nach betriebsfähigen Arbeitsstrukturen waren sachgerechte Arbeitszeiten zu verhandeln, aber auch Räumlichkeiten zu klären, Frauen zu fördern, Organisationsänderungen zu verfolgen sowie die Hauptaufgabengebiete Fallmanagement und Leistungsgewährung personaleinsatzpolitisch abzugrenzen.

Die Interessenvertretungen in den ARGEn bewegen sich im Gegensatz zu ihren Kollegen in den Optionskommunen auf unsicherem Terrain (vgl. Abschnitt 3). Was die Personalräte trotzdem geleistet haben, zeigen auch hier die Felder strategischer Personalratsarbeit (Abbildung 4). Der Blick

\section{Abb. 4: Strategische Personalratsarbeit während der Implemen- tationsphase im Kooperationsmodell - in \% -}

Frage: Wie bedeutsam waren bisher folgende Felder strategischer Personalratsarbeit im Kooperationsmodell?

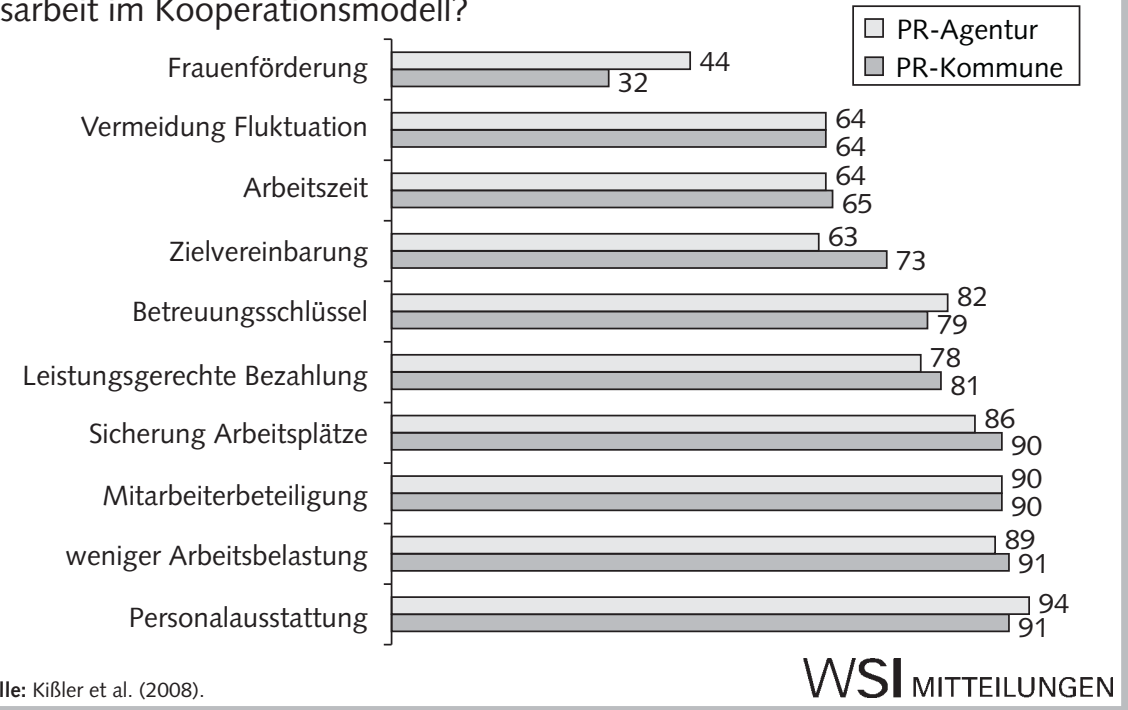

zurück verdeutlicht die Arbeitsschwerpunkte in der Implementationsphase der ARGEn:

Als Folge der Arbeitsverdichtung lag die zentrale Herausforderung der Personalvertretungen vor allem in der Entlastung der Beschäftigten: durch eine bessere Personalausstattung wie einen geringeren Betreuungsschlüssel. Die Arbeitsplatzsicherheit stellte sich aufgrund der Befristungen als weitere Personalvertretungsaufgabe. Die Leistungsgerechtigkeit ist durch eine Diskrepanz zwischen neuen Anforderungen und angemessener Bezahlung ebenfalls zur "tarifpolitischen“ Personalratsaufgabe in den Mischverwaltungen geworden. Ohne die Kompetenz der Beschäftigten kann eine effektive Institutionalisierung der neuen arbeitsmarktpolitischen Institutionen nur schwer gelingen. Dieser Grundsatz wurde in den ARGEn zum Teil missachtet. Daher avancierte auch die Mitarbeiterbeteiligung zu einem interessenvertretungspolitischen Handlungsfeld mit hoher Priorität.

Wie sich Personalratsarbeit in Optionsund Kooperationsmodell künftig gestalten soll, dokumentieren die Befragungsergebnisse zu den perspektivischen Interessenvertretungsstrategien.

In den optierenden Kommunen ( $A b$ bildung 5) hat mittlerweile ein institutioneller Konsolidierungsprozess eingesetzt, der die Schwerpunkte der Personalvertretungen verschieben wird:

Die Personalräte richten weiterhin ihr Augenmerk auf die Verbesserung der Beschäftigtenpartizipation und werden Vereinbarungen abschließen, wie und mit welchen Zielvorgaben die Mitarbeiterinnen und Mitarbeiter geführt werden. Im Rahmen dieser Personalpolitik wollen die Personalräte zudem prüfen, in welchem Gleichoder Ungleichgewicht sich Personalbestand und Fallzahlen einpendeln. Auch die „Tarifverhandlungen“ sind in den Optionskommunen noch nicht abgeschlossen. Die Personalvertretungen werden sich darum bemühen, dass die Arbeitsentgelte (vor allem im Fallmanagement und in der Leistungsgewährung) den neuen arbeitsmarktpolitischen Aufgabenstellungen genügen. Zum weiteren Schutz der Beschäftigten durch den Personalrat gehören, neben ungelösten Problemen mit der räumlichen Unterbringung, die Reduzierung der Arbeitsbelastungen und die Sicherung der Arbeitsplätze, auch um die Fluktuation zu verringern. Die Personalräte wollen sich 
darüber hinaus mehr auf ihre Gestaltungsfunktion bei organisatorischen Innovationen konzentrieren, welche die optierenden Kommunen im Rahmen kontinuierlicher Verbesserungsprozesse planen und umsetzen. Das nachfolgende Ziel der Personalratsarbeit ist daher die Mitgestaltung bzw. Kontrolle von Veränderungen der Binnenorganisation im Zuge der Optimierung des Optionsmodells. Das System von Betreuung und materieller Absicherung ist dagegen funktionsfähig geworden, daher verliert die Abgrenzung von Fallmanagement und Leistungsgewährung in der Personalvertretungsarbeit an Bedeutung. Das gilt im Übrigen auch für die Regelung der Arbeitszeiten.

Trotz problematischer Mitbestimmungsbedingungen justieren auch die „ARGE-Personalräte“ ihre Arbeit, wie der Blick nach vorn in die Interessenvertretungsarbeit im Kooperationsmodell aufzeigt (Abbildung 6).

Die Verringerung der Arbeitsbelastungen und die Mitarbeiterbeteilung bleiben für die Personalräte aus Arbeitsagentur wie Kommune - mit aufstrebender Priorität gleichermaßen wichtige Handlungsfelder. Ein Zukunftsthema ist zudem die Personalausstattung. Signifikant weichen die Zukunftsstrategien der Interessenvertretungen aus unterschiedlichen Herkunftsorganisationen allerdings zur „Arbeitszeit“ ab. Die Personalräte der Agentur erkennen darin eine deutlich größere Herausforderung als ihre kommunalen Kollegen. Die Arbeitszeitgestaltung spielt für die Agenturvertretungen wohl auch deshalb eine größere Rolle, weil die tarifierten Arbeitszeiten der Bundesbeschäftigten länger sind als diejenigen der kommunalen Beschäftigten. Die Anpassung der unterschiedlichen Arbeitszeitregelungen könnte in einer gemeinsamen Einrichtung die wahrgenommenen Ungerechtigkeiten innerhalb der Belegschaft auflösen. Eine deutlich geringere Herausforderung bleibt die Frauenförderung. Sie wird auch in der künftigen strategischen Personalratsarbeit zur nachgeordneten Aufgabe, wenngleich mit mehr Gewicht als zuvor.

Die Personalräte der neuen Arbeitsverwaltungen aus Options- und Kooperationsmodell haben in der bundesweiten Befragung somit ihr Votum zu den Verbesserungen der Arbeitsbedingungen abgegeben. Eine Arbeitspolitik, welche die Arbeitsbelastungen in diesen Institutionen abbaut, wird allerdings kein einfaches Vorhaben sein.
Abb. 5: Strategische Personalratsarbeit der Zukunft im Optionsmodell - in \% -

Frage: Wie bedeutsam sind in der Zukunft folgende Felder strategischer Personalratsarbeit im Optionsmodell?

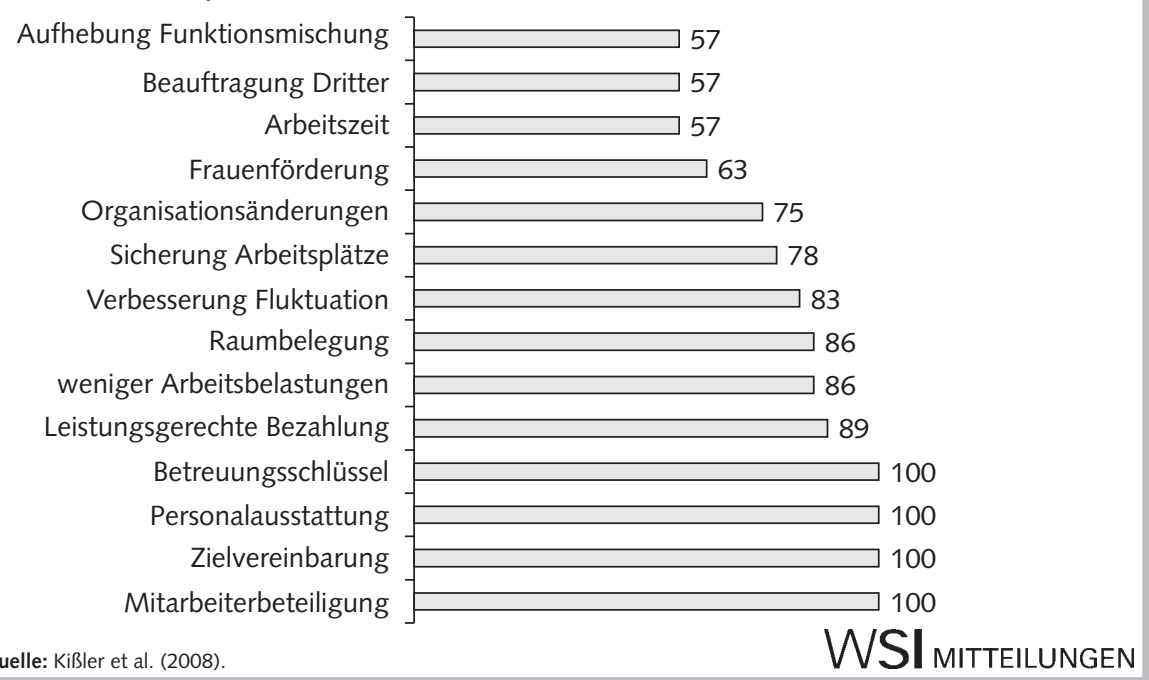

\section{Abb. 6: Strategische Personalratsarbeit der Zukunft im Kooperationsmodell - in \% -}

Frage: Wie bedeutsam sind in der Zukunft folgende Felder strategischer Personalratsarbeit im Kooperationsmodell?

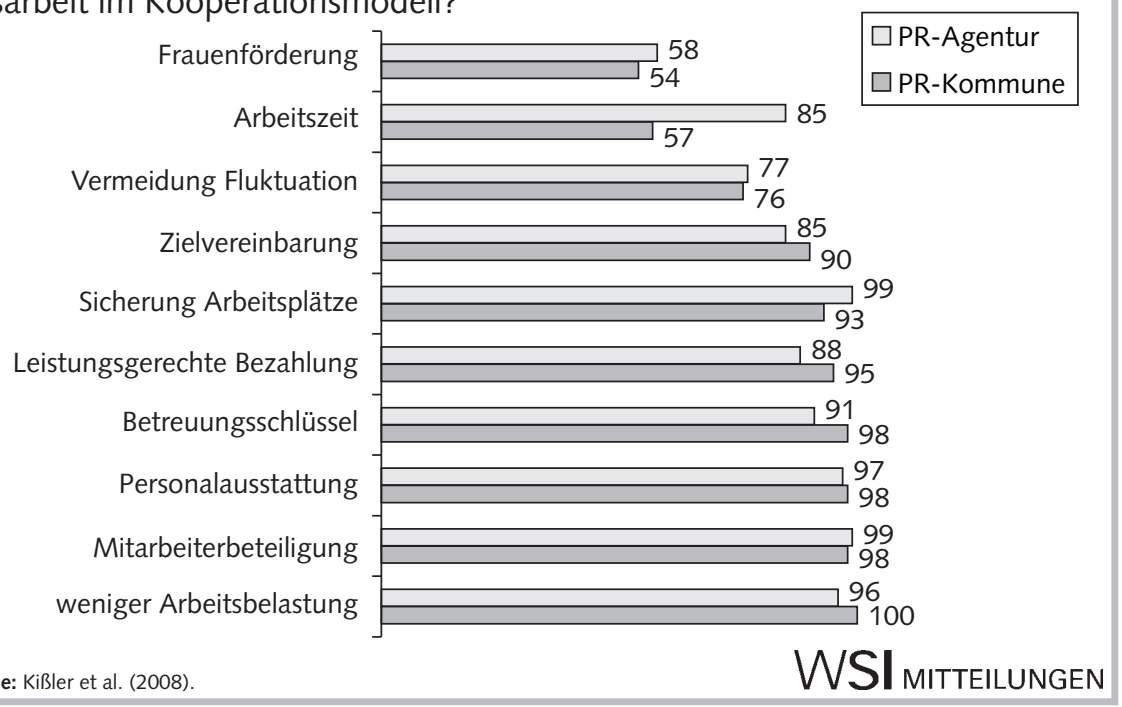

tem Innovations- und Erfolgsdruck stehen? Und sind unter diesen Voraussetzungen gute Arbeitsbedingungen umzusetzen, die sich an den Bedürfnissen der Beschäftigten, ihrem Schutz und dem Erhalt ihrer Arbeitskraft orientieren, aber zugleich eine verbesserte Dienstleistungs- und Servicequalität in Aussicht stellen (vgl. auch Greifenstein/Weber 2007)?

Bewerten wir zunächst die Arbeitsbelastungen.

Die Personalräte aus Kooperationsund Optionsbereich heben die Arbeitsverdichtung als primären Belastungsfaktor die Arbeitsbedingungen der Besc tigten überprüft, muss im Falle dieser Arbeitsmarktreform vornehmlich nachfragen: Wie gestalten sich die Arbeitsbelastungen in einem Organisationsexperiment mit offenem Ausgang, in dem Führungskräfte, Personalräte und Beschäftigte unter erhöh- 
hervor. Dies weist bereits darauf hin, dass alle Vorstellungen von "guter Arbeit" derzeit noch eine eingeschränkte Realisierungschance haben. Arbeitsverdichtung ist allerdings ein durchgreifender Prozess, der bereits im letzten Jahrzehnt im Zuge der deutschlandweiten Rezeption des New Public Managementkonzepts in die Kommunalverwaltungen kam und noch anhält (Kißler 2007, S. 17ff.). Von daher ist es nicht verwunderlich, dass gerade während der Gründung neuer Institutionen dieser Rationalisierungsmainstream der Verwaltungsarbeit für die Beschäftigten kaum günstiger ausfällt. In einer offenen Strukturreform hat dies auffällige Folgen für alle Beschäftigten: Intensivierung der Arbeit, Leistungsdruck und psychische Belastungsvielfalt am Arbeitsplatz charakterisieren die Arbeitsbedingungen in beiden Reformmodellen. Weitere Belastungsfaktoren addieren sich sowohl auf Kooperations- als auch auf Optionsseite hinzu, wie zum Beispiel der zunehmende bürokratische Aufwand, dem die Beschäftigten auf Kosten der Betreuungs- und Beratungstätigkeit im Kundenkontakt ausgesetzt sind. Dazu kommen ungesicherte, befristete Arbeitsverhältnisse. Aber auch die Arbeitskarriere der Festangestellten bei Kommunen oder Agenturen für Arbeit ist keineswegs gesichert, solange nicht feststeht, wie das Organisationsexperiment ausgehen wird.

Die Analysen zu den Arbeitsbedingungen in Options- wie Kooperationsmodellen decken eine weitere Problemzone auf. Der erste Arbeitsmarkt nimmt überwiegend nur das arbeitsmarktnähere Klientel auf, was zur Folge hat, dass die Mitarbeiter/innen des Fallmanagements zwar mit dem Ansatz des „Förderns und Forderns“ antreten, ihn in der Praxis aber nur bedingt umsetzen können. Dieser Kontrast zwischen Anspruch und Wirklichkeit ist eine zusätzliche Arbeitsbelastung. In der Praxis wären die neuen Organisationen und ihre Beschäftigten daher nicht nur an den Vermittlungsquoten zu messen, sondern an ihren gesamten arbeitsmarkt-, sozial- und gesellschaftspolitischen Integrationsstrategien und -leistungen. Nicht ohne Grund wurde bereits in wissenschaftlichen Handlungsempfehlungen zum Umbau der BA festgestellt, dass die geschäftspolitischen Ziele weniger auf kurzfristige Integration, sondern stärker auf die Vermeidung von Langzeitarbeitslosigkeit auszurichten sind und ein überdeterminiertes Controlling zurückgenommen werden sollte (Bender et al. 2006, S. 12). Dieses Vorhaben wird aber nur unter der Voraussetzung gelingen, dass den Beschäftigten ausreichende Freiräume dafür zugestanden werden, was derzeit ein noch zu hoher Betreuungsschlüssel, die Leistungsverdichtung bzw. Arbeitsintensität verhindern. Das hat die Analyse der Arbeitsbelastungen aufgedeckt.

Die Ausprägung von Arbeitsbelastungen zeigt für die Beschäftigten im Kooperations- und Optionsmodell indes auch Unterschiede (vgl. Abb. 1 und 2). Dies betrifft sowohl die hohen Fallzahlen als auch die Betreuung einer marktfernen Klientel und insgesamt die Anforderungen, die sich aus dem Publikumsverkehr ergeben. In all diesen Fällen halten die Personalräte im Optionsmodell die resultierenden Belastungen für die Beschäftigten für geringer als ihre Kollegen in den ARGEn. In zentralen Bereichen wie Fallzahlbearbeitung und Kundenbetreuung unterliegen die Beschäftigten in den Optionseinrichtungen offenbar günstigeren Arbeitsbedingungen als im Kooperationsbereich, d.h. es ergeben sich durchaus Belastungsvorteile für die Beschäftigten im Optionsmodell. ${ }^{2}$

In der Gesamtbilanz zeigt sich: Der Systemversuch impliziert durchaus personalpolitische Botschaften, die zu Existenz, mindestens aber zu Versagensängsten bei den Beschäftigten führen können. Die potenziellen Verschleißkosten von Arbeitskraft liegen damit keineswegs niedrig - und zwar in beiden Modellen. Interessenvertretungsarbeit ist daher in beiden Modellen zu leisten, um belastende Arbeitsbedingungen zu verbessern. Die Personalräte in den Einrichtungen des Optionsmodells sowie in den ARGEn benennen für ihre strategische Interessenvertretungsarbeit dazu durchaus vergleichbare Felder und Prioritäten. Allerdings sind die Voraussetzungen und Chancen unterschiedlich, diese Vorhaben auch umzusetzen.

Die Personalräte aus dem Optionsmodell versuchen im Rahmen eines Ko-Managements, eine effiziente Arbeitsorganisation zu fördern, die gleichzeitig verbesserte Arbeitsbedingungen für die Beschäftigten verspricht. Dieser Weg entspricht vor allem dem Ziel der Personalvertretungen, ihr Optionsmodell über den politisch vorgesehen Zeitraum hinaus dauerhaft als arbeitsmarktpolitische Institution zu etablieren. Diese Absicht haben sie in den Fallstudien mit Nachdruck unterstrichen. In diesem Verständnis werden die Interessen der Beschäftigten vertreten und Vereinbarungen (zu Arbeitsverhältnissen, Personalentwicklungsstrategien etc.) getroffen, die dem Erhalt der neuen Organisationseinheiten dienen sollen. Perspektive ist allerorts das Gelingen des Optionsexperiments und die Verstetigung des Optionsmodells.

Die Personalvertretungsstrategien setzen im Falle des Kooperationsmodells dagegen unverkennbar voraus: Erst wenn die Personalräte abgesicherte Mitbestimmungsrechte erhalten, könnten sie ihre Schutz- und Gestaltungsaufgaben für die Beschäftigten wirksam ausüben. In den ARGEn liegt aber die Mitbestimmung im Argen. Personalräte aus Arbeitsagentur und Kommune stehen vor interessenvertretungspolitischen Hürden, die nur schwer oder auf Umwegen zu überwinden sind. Die interessenvertretungsschwachen Arbeitsbeziehungen wären folglich vorrangig, aber keineswegs ausschließlich im Arbeitnehmerinteresse zu verbessern. Die Kompetenz von Personalvertretungen als KoManager ist auch in den ARGEn zu nutzen, einmal mehr angesichts der ungesicherten Beschäftigungsperspektive der Mitarbeiterinnen und Mitarbeiter und der ungeklärten Aussicht, unter welchen institutionellen Bedingungen sie künftig tätig werden sollen. Erste Evaluationen der GovernanceStrukturen zeigen inzwischen, dass die Optionskommunen einen größeren operativen und strategischen Handlungsspielraum haben, während die ARGEn durch komplexe Aushandlungen, geringe dezentrale Entscheidungsbefugnisse und hohe Rechtsunsicherheit geprägt sind (Kaps et al. 2008). Nach den vorgestellten Ergebnissen unserer Studie gilt Vergleichbares auch für die Interessenvertretung in den neuen Arbeitsverwaltungen, trotz der unterschiedlichen Versuche, alternative Formen der Interessenregulierung in den ARGEn zu etablieren.

Für Beschäftigte und ihre Personalräte scheint im Options- wie Kooperationsmodell zusammenfassend zu gelten: Belastende Arbeitsbedingungen sind in beiden Fällen auch Folge der institutionellen Unsicherheit bzw. Rückholbarkeit. Die Voraussetzungen, „Gute Arbeit“ umzusetzen, sind schwierig und unterscheiden sich zwischen

2 Allerdings ist bei diesem Ergebnis Vorsicht geboten. Es handelt sich um Einschätzungen der Arbeitsbedingungen durch die befragten Personalräte, nicht jedoch um „objektiv“ gemessene Belastungsfaktoren. 
Options- und Kooperationsmodell, da die Mitbestimmungsstrukturen in den ARGEn brüchig sind. Der Aufschlag zu einer erneuten verbands- und parteipolitischen Diskussion über die institutionelle Arbeitsmarktreform liegt inzwischen mit dem Konzept des „Kooperativen Job-Center“ vor. Ob diese abgeschwächte Variante der ARGE die Kooperationsschwierigkeiten und arbeitspolitischen Rahmenbedingungen verbessern würde, bleibt abzuwarten. Auch die 19 Kommunen mit getrennter Aufgabenwahrnehmung sind sicher kein Königsweg, denn sie belassen durch das zwischen BA und Kommunen gespaltene „Fördern und Fordern“ bei der Betreuung der Arbeit suchenden Klientel alles beim Alten. Vor einem politischen Schnellschuss müssen alle Konzepte anhand des noch laufenden Evaluationsprogramms geprüft werden, aber auch mit Blick auf die Arbeitsbedingungen der Beschäftigten und ihre Chancen zur vernünftigen Interessenregulierung. Das ist die arbeitspolitische Schattenseite und zugleich Herausforderung dieser institutionellen Strukturreform mit offener Zukunftsarchitektur.

\section{LITERATUR}

Bender,G./Bieber, D./Hielscher, V./Marschall, J./Ochs, P./Vaut, S.

(2006): Organisatorischer Umbau der Bundesagentur für Arbeit. Evaluation der Maßnahmen zur Umsetzung der Vorschläge der Hartz-Kommission, Kurzfassung, ISO-Institut Saarbrücken

Bertelsmann Stiftung (2006): Jahresbericht 2006 für das SGB-II-Benchmarking der Optionskommunen, Gütersloh

Greifenstein, R./Weber, H. (2007): Vom Klassiker "Humanisierung der Arbeit" zum Zukunftsprogramm "Gute Arbeit", Schriftenreihe der Friedrich-Ebert-Stiftung „WISO direkt" , September, Bonn

Institut für Sozialforschung und Gesellschaftspolitik (ISG) (2007): Jahresbericht 2007. Evaluation der Experimentierklausel nach § 6c SGB II Vergleichende Evaluation des arbeitsmarktpolitischen Erfolgs der Modelle der Aufgabenwahrnehmung "zugelassene kommunale Träger" und „Arbeitsgemeinschaften ", Köln
Kaps, P./Oschmiansky, F./Mosley, H. (2008): Experimenteller Wettbewerb. Evaluation zeigt problematische Strukturen bei Hartz IV, in: WZBMitteilungen 119, S. 35-37

Kißler, L./Greifenstein, R./Wiechmann, E. (2008): Großbaustelle Arbeitsverwaltung. Arbeitsbedingungen und -beziehungen im Schatten der Arbeitsmarktreform. Modernisierung des öffentlichen Sektors, Sonderband 31, Berlin

Kißler, L. (2007): Warum die kommunale Verwaltungsreform (fast) gescheitert ist oder: Wo bleibt die "Reformdividende" für die Beschäftigten?, in: Bogumil, J./Holtkamp, L./Kißler, L./Kuhlmann, S./Reichard, Ch./Schneider, K./Wollmann, H.: Perspektiven der kommunalen Verwaltungsreform, Berlin, S. 17-26

Knuth, M./Koch, F./Schweer, O. (2007): Kommunalisierte Grundsicherung für Arbeitsuchende. Abschlussbericht, Hans-Böckler-Stiftung, Düsseldorf 


\section{ZUSAMMENFASSUNGEN}

\section{GREIFENSTEIN, RALF/KIßLER, LEO/WIECHMANN, ELKE}

Arbeitsbedingungen und Mitbestimmung in den neuen

Arbeitsverwaltungen

in: WSI-Mitteilungen 9/2008, Seiten

Die institutionelle Reform der Arbeitsmarktpolitik führt zu beachtlichen Arbeitsbelastungen sowohl im Kooperationsmodell zwischen Bundesagentur für Arbeit und Kommune wie auch im Optionsmodell mit kommunaler Alleinträgerschaft. Diese Arbeitsbedingungen fordern die Mitbestimmung der Personalvertretungen. Die Personalräte aus Kommune und Arbeitsagentur bewegen sich in den Mischverwaltungen des Kooperationsmodells aber in experimentellen Mitbestimmungsstrukturen, wodurch ihre Verhandlungsmacht und ihre Chancen sinken, die Arbeitsbedingungen zu verbessern. In den optierenden Kommunen greift zwar eine erprobte und rechtlich abgesicherte Mitbestimmung, aber auch in den neuen kommunalen Organisationseinheiten akzeptieren die Beschäftigten und ihre Personalräte belastende Arbeit, um dieses Modell langfristig zu konsolidieren. Allerdings sind die institutionellen Perspektiven nach dem jüngsten Urteil des Bundesverfassungsgerichts für alle Beschäftigten in Options- wie Kooperationsmodell gleichermaßen ungesichert. Die Schattenseite dieser Arbeitsmarktreform sind somit die eingeengten Erfolgsaussichten, "Gute Arbeit" für die Beschäftigten in den neuen Arbeitsverwaltungen umzusetzen. 